\title{
Interpretación causal, sentimiento de culpa y predicción de reincidencia en un contexto legal
}

\author{
Ana Rosa Delgado González \\ Universidad de Salamanca
}

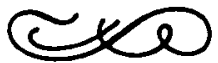

\section{Resumen}

Hemos realizado un estudio diferencial sobre las interpretaciones causales y sus consecuencias cognitivo. afectivas en un contexto de robo, utilizando como marco teórico el último modelo atribucional de Weiner (1986). Para ello, hemos aplicado cuestionarios de autoinforme a 1.450 sujetos menores de edad. Los resultados, obtenidos mediante análisis de la varianza y técnicas de regresión, indican (1) que existe una taxonomia de las causas claramente diferenciada en función de las dimensiones -locus, estabilidad y controlabilidad-, y de la perspectiva - actor/observador - de los sujetos; (2) que la estabilidad de las causas determina la predicción de reincidencia, y que ésta es mayor en los sujetos que dicen baber cometido más robos, y (3) que el sentimiento de culpa está determinado por la controlabilidad de las causas y varía como función inversa de la experiencia delictiva. Finalmente, señalamos algunas implicaciones teóricas y aplicadas de este trabajo.

\section{Abstract}

A differential researcb bas been carried on causal interpretation and its cognitive-afective consequences in the context of theft, using Weiner's last attributional model (1986) as a theoretical background. Questionnaires of self-report were answered for 1450 minors. The results, obtained by means of ANOVA and regression methods, show that (1) there is a differential causal taxonomy depending on causal dimensions - locus, stability and controllability-, and subjects actorlobserver perspective; (2) that causal stability determines prediction of recidivism, which is greater for self-declared delinquents; and (3) that feelings of guilt are predicted by causal controllability and vary inversely with criminal experience. Some theoretical and applied implications of the present work are discussed finally.

Agradecimientos: La investigación que ha dado lugar a este trabajo está financiada por una beca del Plan de Formación de Personal Investigador en su línea complementaria de Criminología, desviación social y rehabilitación.

Dirección del autor: Departamento de Psicología, Avda. de la Merced s/n, 37005 Salamanca. 


\section{INTRODUCCION}

Una de las consecuencias del aprendizaje de las normas sociales es la regulación de la conducta mediante reacciones de autocondena (Bandura y McDonald, 1963), entre las que se encuentra el sentimiento de culpa. De éste se ha dicho que ocurre en situaciones en que uno se siente personalmente responsable (Izard, 1977) que respecto de él el sí mismo acepta la responsabilidad por una violación del orden moral (DeRivera, 1984) y que lo experimentamos cuando somos personalmente responsables de resultados negativos que violan las normas sociales o morales (McGraw, 1987).

En la intersección entre el estudio del juicio moral y el de las emociones podemos situar los sentimientos de culpa, vergüenza, orgullo y otros afectos en los que la dimensión evaluativa - moral y/o social- es innegable, razón por la cual resultan de particular interés para el estudio de la conducta desviada. Una característica distintiva de la mayor parte de la conducta humana es que se halla gobernada por reglas (Sabini y Silver, 1982; Ardila, 1986) y existe evidencia de que éstas, y por tanto aquélla, dependen del contexto social en que se apliquen. Los juicios morales, en la práctica, son decisiones que tienen que ver con dos o más reglas morales conflictivas aplicables a una situación particular (Kurtines, 1986).

Esta nueva orientación ha afectado al estudio de las emociones, tradicionalmente tan alejado de los esquemas sociales, que ha dejado a un lado el misticismo de la inefabilidad (Frijda, 1988) y se ha dedicado a estudiar las leyes que conectan las estructuras de significado con las emociones y con los sentimientos, los grandes marginados de la orientación naturalista. Se postula que la emoción envuelve valores sociales internalizados que hacen que el agente capaz de sentir la emoción apropiada se adhiera autónoma y fiablemente a los valores que esa emoción marca (Armon-Jones, 1986), lo cual no es sino la otra cara de la moneda del desarrollo moral. Así, sentimientos como el de culpa cumplen una función social tan importante como es preservar las reglas del grupo, aunque no se niegue la posibilidad de que existan respuestas emocionales previas a la socialización o asociadas a conductas más o menos alejadas de ésta (que son las que tradicionalmente se estudian bajo el epígrafe de emociones, p.e. Izard y Saxton, 1988).

Todo esto implica, además, que estados tradicionalmente considerados como producto de un defecto madurativo - la carecia de sentimiento de culpa en los denominados psicópatas o la irresponsabilidad de los menores, por ejemplopodrían no ser productos naturales, sino más bien estados generados socialmente.

Crespo (1986), refiriéndose a la existencia de un doble código moral y conductual en los niños, observa que cuando se les exige algo firmemente muestran una especie de infantilización caracterizada por conductas y sentimientos ingenuos que los adultos consideran apropiados en la infancia; sin embargo, también poseen la capacidad de actuar sobre la base de esquemas adultos en el grupo de iguales o en otras situaciones que así lo requieran.

Weiner (1986), cuya teoría atribucional ha dedicado un lugar central a las emociones en los últimos años, considera, como los anteriores, que la situación determina qué emociones van a ser experimentadas. Pero además especifica cómo: es la interpretación de las situaciones en términos de dimensiones causales la que determina lo que sentimos. Sus investigaciones documentan que al menos cinco emociones humanas prevalecientes, la ira, la pena, la culpa, la grati- 
tud y la vergüenza, están determinadas por las percepciones de causalidad. Más específicamente, esas emociones se guían por las percepciones de control personal y de responsabilidad (Weiner, 1987).

Shaver (1985) ha buscado apoyo empírico para un modelo dimensionalnormativo de la responsabilidad que cuenta a su favor con una extensa revisión del significado de los conceptos, refinado filosóficamente durante siglos. Shaver, Null y Huff (1982, citado en Shaver, 1985) realizaron un escalamiento multidimensional sobre 18 palabras relacionadas con la responsabilidad y hallaron que los adjetivos intentado y previsible prácticamente ocupaban la misma posición en el espacio bidimensional junto a controlable. Dada la similitud de significados parece que, para dar cuenta de la atribución de responsabilidad del lego, no es necesario tener en cuenta la diferenciación normativa entre tales dimensiones.

Weiner, Graham y Chandler (1982) pidieron a sus sujetos que recordasen emociones y encontraron que el $94 \%$ de las causas asociadas con sentimientos de culpa se consideraban internas y controlables, es decir, el tipo de causas por las que, normativamente, uno debería asumir la responsabilidad. El modelo de Weiner (1986), anclado en la causalidad percibida - es decir, en la causalidad tal como se entiende en la calle y en la ley-, considera que la culpa se experimenta a continuación de un acto cuya causa se interpreta en términos de causalidad interna y controlabilidad. Las investigaciones de Carroll $(1978,1979)$ y Carroll y Payne (1976, 1977a, 1977b) habían adaptado un modelo anterior, en el que no se tenían en cuenta las emociones, al contexto legal de la predicción de reincidencia. Basándonos en ambos acercamientos, y utilizando como contexto la violación de una norma legal, hemos construido un marco teórico y metodológico con el que investigar si la interpretación dimensional de la causalidad trae consigo realmente esas consecuencias cognitivo-afectivas.

Nuestro trabajo podría haber tomado como modelo una investigación que situase el origen de la secuencia en la emoción o en cualquier otro de sus elementos, pero, dada la escasez de trabajos al respecto, hemos preférido adaptarnos lo más fielmente posible a las hipótesis del modelo de Weiner (1986) de modo que éste, caracterizado por más de veinticinco años de continuadas inves-

\section{FIGURA 1}

Relaciones bipotetizadas por el modelo (basado en Carroll y Payne, 1977 a y Weiner, 1986).

Antecedentes Dimensions de las causas

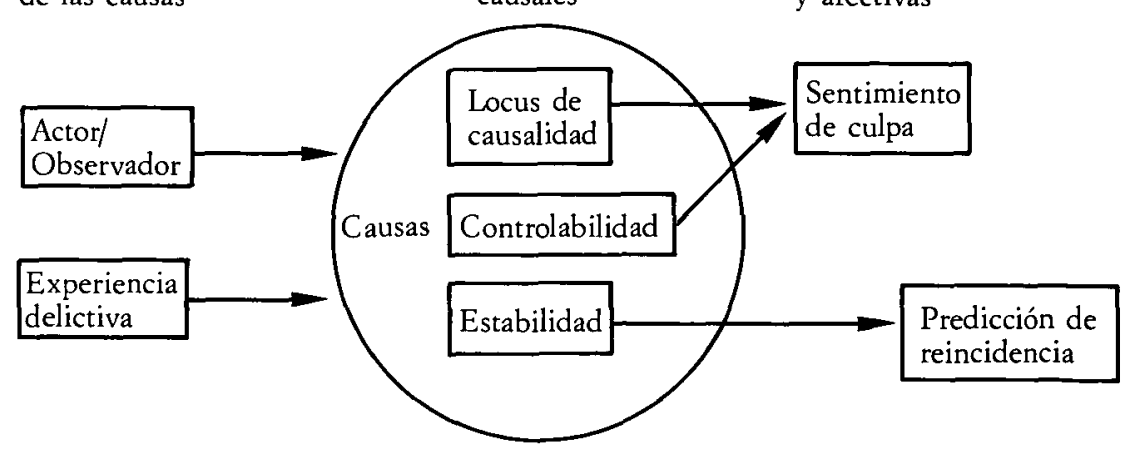




\section{4}

tigaciones empíricas, pudiera servirnos de guía en la búsqueda de materiales, en los procedimientos y en otras cuestiones prácticas.

La experiencia delictiva - una variable de la muestra - y la perspectiva - una variable de la tarea - se incluyeron en la investigación por razones de diferente rango. La primera, relativa a la aplicación de este tipo de investigaciones, tiene que ver con la exploración de diferencias atribucionales y/o emocionales potencialmente criminógenas, mientras que la segunda, de carácter teórico, está en relación con los dos paradigmas que se suelen utilizar en la operacionalización de las variables atribucionales, sobre la que probablemente esté actuando el clásico efecto de la diferencia entre actor y observador (Jones y Nisbett, 1972).

Primer problema: ¿Cómo se relacionan las causas que nos dan los sujetos con las dimensiones causales de locus, estabilidad y controlabilidad?

Segundo problema: La interpretación dimensional de las causas del delito ¿está relacionada con la predicción de reincidencia y el sentimiento de culpa, tanto cuando uno se juzga a sí mismo como cuando juzga a los demás e independientemente de la experiencia delictiva de quien juzga?

Hipótesis 2.1: La predicción de reincidencia es una función de la dimensión subjetiva de estabilidad.

Hipótesis 2.2: El sentimiento de culpa es una función de las dimensiones subjetivas de locus de causalidad y controlabilidad.

Hipótesis 2.3: Las relaciones hipotetizadas más arriba no son independientes de la experiencia delictiva de quien juzga.

Hipótesis 2.4: Las relaciones hipotetizadas más arriba no son independientes de la perspectiva (actor/observador) de quien juzga.

\section{METODO}

\section{Muestra}

La muestra inicial fue de 1.450 sujetos, práticamente todos los alumnos que cursaban $8 .^{\circ}$ de EGB en los meses de abril y mayo de 1989 en los 28 colegios públicos de la ciudad de Salamanca.

De la muestra original se eliminaron 82 sujetos con puntuaciones inferiores a 4 en la escala $S$ de deseabilidad social del E.P.I. (forma A de la adaptación española), por lo que la muestra considerada finalmente fue de 1.368 sujetos con edades comprendidas entre los 13 y los 16 años.

Elegimos esta muestra por tratarse específicamente de los sujetos con edades conflictivas desde el punto de vista legal, ya que, aunque se les considere inimputables, pueden ser sancionados por los tribunales tutelares de menores, en ocasiones duramente, si cometen faltas o delitos, y esto último resulta muy común en el momento presente. Por otra parte, desde el punto de vista de la intervención, el caso de los menores cuenta a su favor con una concepción proteccionista y educativa muy respaldada socialmente, por lo que las investigaciones aplicadas suelen resultar más fructíferas que en el caso de sujetos adultos.

\section{Procedimiento}

En el contexto de una investigación más amplia, realizada mediante cuestionario, los sujetos respondieron a preguntas relativas a las atribuciones causales sobre el robo y a su experiencia delictiva específica. Hemos utilizado como 
contexto legal un único delito, el de hurto, que los datos del Tribunal Tutelar de Menores señalan como la infracción más extendida entre los menores de edad.

Las atribuciones causales se realizaron en dos tipos de tarea en los que el sujeto debía responder previamente a la cuestión por qué.

En la tarea heteroatribucional se utilizó un paradigma de juicio social; los sujetos leyeron una breve descripción de un robo: alguien de tu clase ba robado 30.000 pts. Posteriormente los sujetos respondieron a la Escala de Dimensiones Causales (Russell, 1982) que evalúa las dimensiones subjetivas de locus de causalidad, estabilidad y controlabilidad en situaciones específicas mediante nueve escalas de diferencial semántico. Por último, emitieron juicios relativos a la intesidad del sentimiento de culpa que se cree ha experimentado el agente del robo y a la probabilidad de comisión de ese delito u otro similar en el futuro. Tanto éstos como aquéllas fueron valorados por el sujeto mediante puntuaciones de 0 a 10 que señalaban su intensidad. La utilización de esta escala a lo largo de toda la prueba se debe a la familiaridad de los escolares con las notas.

Puesto que la descripción no ofrece información suficiente, los sujetos responderán a la pregunta por qué según sus creencias al respecto o, en términos atribucionales, según su esquema causal (Kelley, 1972). Las relaciones entre las variables en este tipo de tarea nos indicarán la teoría implícita que sobre el robo y sus consecuencias tienen los sujetos (Moreno-Jiménez, 1986).

Para la tarea autoatribucional utilizamos un paradigma de tipo retrospectivo (Weiner, Russell y Lerman, 1979), tradicionalmente empleado en estudios sobre la emoción, en el que se pidió a los sujetos que recordaran y describieran brevemente una situación en la que fueron ellos los agentes de robo. La descripción se solicitó con el fin de evaluar la gravedad de los hechos y eliminar aquellos casos en los que lo que se describía era más bien un hallazgo fortuito, un préstamo o cualquier otro suceso no clasificable como hurto o robo.

A partir de ahí la tarea es formalmente idéntica a la anterior excepto en el referente de los juicios, ya que en este caso el agente es el propio sujeto.

Somos conscientes de que este procedimiento de recuerdo se enfrenta con la posibilidad de diversos sesgos de respuesta, nociones estereotipadas o estrategias defensivas. Pero resulta difícil imaginar que el recuerdo que la gente tiene de sus emociones no esté en absoluto relacionado con la naturaleza de los procesos emocionales reales. Puesto que el sentimiento subjetivo sólo puede conocerse preguntando directamente a los sujetos, éste es, por el momento, el procedimiento más adecuado para llegar a la naturaleza de las emociones cotidianas; sólo a partir de los patrones obtenidos mediante autoinforme será posible utilizar paradigmas experimentales con mayor validez ecológica (Scherer y Tannenbaum, 1986).

La experiencia delictiva específica se operacionalizó mediante la escala de robo del Cuestionario de conductas antisociales, construido por Luengo, Mirón y Otero (Mirón, 1989-comucicación personal, Mirón, Otero y Luengo, 1989), y dirigido a sujetos entre 11 y 18 años, que consta de 18 ítems y posee una eleva$\mathrm{da}$ consistencia interna (alfa $=.95$ ).

\section{RESULTADOS}

Todos los análisis se han realizado mediante el paquete estadístico SPSS/ PC + para IBM PC/XT/AT (1985). 
Las causas se codificaron, tras una primera revisión exhaustiva, en más de cincuenta categorías, con el fin de no desechar en un primer paso información que pudiera ser relevante desde el punto de vista cualitativo. Posteriormente, puesto que la mayoría de las causas compartían características que permitían una agrupación lógica en un menor número de categorías, se realizó una recodificación que resultó más sencilla en la medida en que muchas de las categorías del primer código contaban con porcentajes inferiores al $1 \%$ de los casos y pudieron reunirse bajo el epígrafe genérico de otras. El código resultante incluyó las siguientes categorías: deseo, amistades, problema, presumir, drogas, fastidiar, vicio, necesidad, necesidad familiar, tipo de persona y situación.

Por otra parte, clasificamos según su gravedad los hurtos y robos descritos en la tarea autoatribucional con el fin de ver si existía, a priori, una diferencia fundamental entre los dos paradigmas de tarea. Los episodios descritos por los sujetos se codificaron como leves, medios o graves y se realizaron distintas pruebas paramétricas y no paramétricas para explorar la existencia de diferencias de medias en las distintas variables del modelo en función de la gravedad de los episodios descritos. Aunque la mayoría de los episodios descritos se clasificaron como leves, no encontramos efectos significativos de la variable gravedad, por lo que condideramos que es posible, en general, tomar ambas tareas como equivalentes, excepto en lo referente a la perspectiva.

Por último, la Escala de Dimensiones Causales (Russell, 1982) había mostrado su validez en diferentes investigaciones relacionadas con el logro (Russell, McAuley y Tarico, 1987) y venía avalada por la recomendación del propio Weiner (1986); sin embargo, no había sido utilizada ni en el contexto legal ni en ninguna investigación española —aunque sí se había explorado la existencia de estas dimensiones con resultados negativos (Salgado, 1987)—, razón por la cual realizamos algunos análisis previos para comprobar si los nueve ítems que la componen reflejaban, o no, dimensiones coherentes.

Los análisis factoriales realizados sobre las puntuaciones de la escala en ambas tareas confirmaron la existencia de tres factores y nos indicaron que tan sólo los ítems relativos a la estabilidad resultaban coherentes en ambas tareas. La dimensión de locus y la de controlabilidad eran inadecuadamente medidas por un ítem diferente en cada tarea, lo cual nos llevó a eliminar estos ítems - de pobre discriminación y con pesos factoriales inferiores a .5 en su factor- con el fin de maximizar el valor predictivo del modelo.

\section{Interpretación dimensional de las causas}

Tratamos de realizar, explorando las diferencias significativas entre las medias de cada causa en las tres dimensiones, una taxonomía de las causas del delito similar a la realizada en contextos de logro. No se incluyeron en los análisis aquellas con frecuencia absoluta menor de 30 , que se habían acumulado en una categoría genérica.

Utilizamos el análisis paramétrico de la varianza de un factor cuando no parecían violarse los supuestos y la prueba no paramétrica equivalente, KruskalWallis, cuando alguno de los coeficientes utilizados por la prueba paramétrica ( $\mathrm{C}$ de Cochran y $\mathrm{F}$ de Bartlett-Box) indicaba que podría estarse produciendo dicha violación.

De este modo encontramos que existían diferencias significativas al $1 \%$ en las medias de locus $(\mathrm{F}=13.853, \mathrm{p}=0.0 /$ chi-cuadrado corregido $=77.77, \mathrm{p}=.000)$ 
y estabilidad $(F=20.249, p=0.0 /$ chi-cuadrado corregido $=112.815, p=.000)$ de las diferentes causas de la tarea heteroatribucional; no ocurrió así para la dimensión de controlabilidad $(\mathrm{F}=1.745, \mathrm{p}=.1072)$

En cuando a la tarea autoatribucional, sólo hallamos diferencias significativas al $1 \%$ entre las medias para la dimensión de locus $(\mathrm{F}=10.985, \mathrm{p}=0.0 / \mathrm{chi}$ cuadrado corregido $=40.149, \mathrm{p}=.000$ ).

\section{TABLA I}

Puntuaciones medias de las dimensiones para cada una de las causas del robo en las tareas betero y autoatribucional. Puntuaciones elevadas indican mayor internalidad, estabilidad y controlabilidad en la interpretación de las causas.

\begin{tabular}{lccc}
\hline Causas hetero & Locus & Estabilidad & Controlabilidad \\
\hline Deseo & 6.618 & 3.988 & 6.407 \\
Problema & 5.533 & 3.367 & 6.879 \\
Drogas & 6.726 & 5.411 & 5.715 \\
Vicio & 7.109 & 5.402 & 6.227 \\
Necesidad & 5.577 & 3.675 & 6.163 \\
Nec. familiar & 4.157 & 2.929 & 6.226 \\
Tipo persona & 7.091 & 5.549 & 6.121 \\
\hline Causas auto & Locus & Estabilidad & Controlabilidad \\
\hline Deseo & 5.790 & 1.791 & 5.301 \\
Amistades & 3.917 & 1.588 & 5.912 \\
Fastidiar & 4.627 & 1.371 & 5.183 \\
Necesidad & 4.939 & 1.671 & 4.668 \\
Situación & 4.700 & 1.414 & 4.931 \\
\hline
\end{tabular}

No resultaron significativas ni las diferencias de medias en estabilidad $(\mathrm{F}=.878, \mathrm{p}=.477 /$ chi-cuadrado corregido $=2.787, \mathrm{p}=.594)$ ni las diferencias de medias en controlabilidad $(\mathrm{F}=2.726, \mathrm{p}=.028 /$ chi-cuadrado corregido $=10.628, \mathrm{p}=.031$ ). Esta última dimensión sí resulta significativa al $5 \%$.

\section{TABLA II}

Taxonomia dimensional de las causas del delito en las tareas betero y autoatribucional.

\begin{tabular}{lccc}
\hline & & \multicolumn{2}{c}{ CONTROLABLES } \\
\cline { 3 - 4 } & EXTERNAS & Nec. familiar & - \\
HETERO & INESTABLES & ESTABLES \\
\hline INTERNAS & $\begin{array}{c}\text { Deseo } \\
\text { Necesidad }\end{array}$ & $\begin{array}{c}\text { Vicio } \\
\text { Drogas } \\
\text { Tipo persona }\end{array}$ \\
\hline EUTO & EXTERNAS & $\begin{array}{c}\text { Amistades } \\
\text { Fastidiar } \\
\text { Necesidad }\end{array}$ & - \\
\hline
\end{tabular}


Una vez establecida la significatividad de las diferencias en internalidad y estabilidad, se exploró su ubicación concreta mediante la prueba $\mathrm{t}$ de Scheffe al $5 \%$, una prueba conservadora para la comparación de medias dos a dos, que requiere diferencias entre medias más elevadas que el resto de las pruebas disponibles para comparar medias. La clasificación resultante, en función de las dimensiones de la causalidad y la perspectiva, puede verse en la Tabla 2.

La prueba t de Scheffe al $5 \%$ no encontró diferencias significativas en controlabilidad en la tarea de autoatribución, por lo que se considera que todas las causas del robo ofrecidas por los sujetos en ambas tareas son percibidas o conceptualizadas como tendentes a la controlabilidad, por el sujeto o por otros, dependiendo de si se considera interna o externa, respectivamente.

Podemos observar que la respuesta problema no aparece en esta taxonomía dimensional, ya que las pruebas realizadas no arrojaron diferencias significativas entre ésta y el resto en locus de causalidad, debido probablemente a que había sido la respuesta dada a la tarea heteroatribucional por un menor número de sujetos $(n=30)$ con una gran dispersión en locus. Sabemos, sin embargo, que sí resulta significativamente diferente del resto en estabilidad, situándose en el polo de la inestabilidad.

Lo mismo ocurre con respecto a la respuesta situación de la tarea autoatribucional con el mismo número de sujetos $(n=30)$ que la anterior, muy pequeño en comparación con el resto de las respuestas.

\section{Relaciones atribucionales del modelo.}

Con el fin de responder a la pregunta relativa al segundo problema, calculamos las ecuaciones de regresión correspondientes a las tres primeras hipótesis para las dos tareas, con lo que, simultáneamente, fuimos poniendo a prueba la cuarta hipótesis. Sólo una de las razones $\mathrm{F}$ asociadas a las ecuaciones de regresión resultó significativa únicamente al $5 \%$, por lo que aceptamos con reservas la hipótesis correspondiente. El resto de las razones $\mathrm{F}$ tuvieron probabilidades asociadas de .000 y 0.0 , es decir, las ecuaciones pueden considerarse explicativas con un gran margen de seguridad.

Hipótesis 2.1: La predicción de reincidencia es una función de la dimensión subjetiva de estabilidad.

\section{FIGURA 2}

Regresión de la dimensión causal de estabilidad en las tareas betero (ESTH) y autoatribucio. nal (ESTA) sobre la predicción de reincidencia en ambas tareas (PREH y PREA, respectivamente).

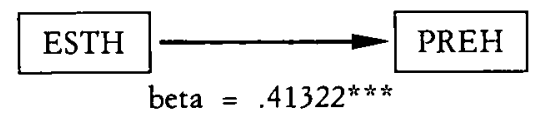

$\mathrm{R}$ cuadrado $=.17012$

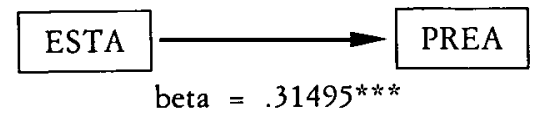

$\mathrm{R}$ cuadrado $=.09846$

El coeficiente de determinación asociado a la ecuación de la estabilidad sobre la predicción de reincidencia de otro fue el más elevado de los obtenidos con respecto al modelo formal. La estabilidad percibida en las causas de la conducta delictiva de otro sujeto explicó un $17 \%$ de la varianza de la predicción 
de reincidencia con respecto a éste. El parámetro beta, indicativo de la cuantía de la relación fue también muy elevado (.41322) y significativo por encima del uno por mil.

Aunque menores, las relaciones entre variables en la tarea atribucional fueron las mismas, por lo que aceptamos esta hipótesis con independecia de la tarea.

Hipótesis 2.2: El sentimiento de culpa es una función de las dimensiones subjetivas de locus de causalidad y controlabilidad.

\section{FIGURA 3}

Regresión de las dimensiones causales de locus y controlabilidad en las tareas betero ( $L H$ y COH) y autoatribucional ( $L A$ y COA) sobre el sentimiento de culpabilidad en ambas tareas (SENTH y SENTA, respectivamente).

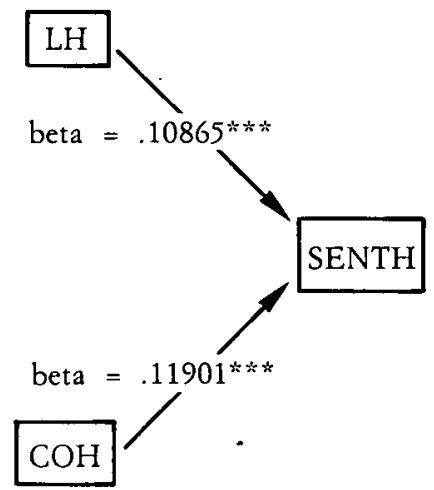

$\mathrm{R}$ cuadrado $=.02574$

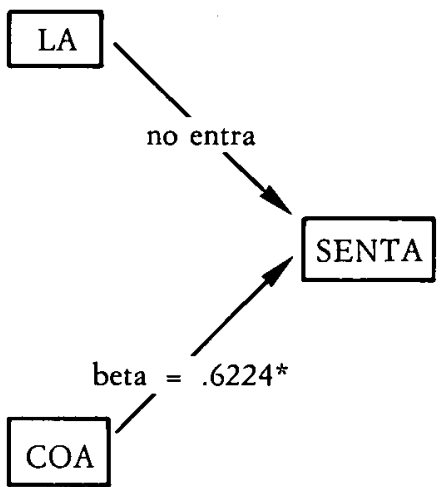

$\mathrm{R}$ cuadrado $=.00305$

Obtuvimos, para ambas ecuaciones, coeficientes de determinación muy pequeños. Esto resultó especialmente cierto en el caso de la tarea autoatribucional, en la que la controlabilidad no llegaba a explicar el $1 \%$ de la varianza del sentimiento de culpabilidad y la ecuación sólo pudo aceptarse como significativa al .05 .

Tampoco pudo aceptarse para ambas tareas la hipótesis sobre la experiencia de culpabilidad. Podemos decir que resultó cierta cuando se trataba de juzgar a otros; pero, cuando los sujetos hubieron de juzgarse a sí mismos, la única dimensión explicativa fue la controlabilidad, que dio cuenta de una pequeñísima parte de la varianza. Puesto que la probabilidad asociada fue de .03 , sólo podríamos aceptarla con un nivel de confianza de .05 .

Por otra parte, la relación resultó ser opuesta en ambas tareas: la dimensión heteroatribucional de controlabilidad se relacionó negativamente con el sentimiento de culpa supuesto en el otro, pero la dimensión autoatribucional de control predijo positivamente la experiencia de culpa.

Hipótesis 2.3: Las relaciones hipotetizadas más arriba son independientes de la experiencia delictiva de quien juzga. 


\section{0}

Respecto de la tercera hipótesis, trabajamos sobre las ecuaciones calculadas en el paso anterior introduciendo al variable implicada - la experiencia delictiva - y utilizando el método de eliminación hacia atrás para comprobar si permanecía en la ecuación, es decir, para comprobar si realmente servía para predecir los criterios, o más bien lo que ocurría era que no tenía ningún peso en la explicación de la varianza de la variable dependiente de la ecuación y, por tanto, era eliminada.

Las ecuaciones no resultaron modificadas en un sentido cualitativo, puesto que las relaciones constatadas en las hipótesis anteriores se mantuvieron constantes. Sin embargo, la experiencia delictiva introdujo cambios significativos -aunque pequeños - en el coeficiente de determinación de tres de las ecuaciones, como puede comprobarse en la Tabla 3.

\section{TABLA III}

Parámetros beta con los que se introduce la variable relativa a la experiencia delictiva especifica (C.A.R.) en las ecuaciones específicas del modelo y coeficientes de determinación de las dos ecuaciones antes y después en ambas tareas.

\begin{tabular}{lcrc}
\hline \multicolumn{1}{c}{ Ecuación } & $\begin{array}{c}\text { R cuadrado } \\
\text { antes }\end{array}$ & beta & $\begin{array}{c}\text { R cuadrado } \\
\text { después }\end{array}$ \\
\hline $\begin{array}{l}\text { LH, COH sobre SENTH } \\
\text { ESTH sobre PREDH }\end{array}$ & .17012 & $\begin{array}{r}\text { no entra } \\
.07566^{* *}\end{array}$ & .17617 \\
COA sobre SENTA & .00305 & $-.06651^{*}$ & .00683 \\
ESTA sobre PREDA & .09846 & $.08616^{* *}$ & .10697 \\
\hline
\end{tabular}

Los resultados indican que la variable de experiencia delictiva específica entra a formar parte de las ecuaciones correspondientes a la predicción de reincidencia en ambas tareas, lo que puede interpretarse en el sentido de los sujetos que más han robado esperan - de sí mismos y de los demás- mayor reincidencia que los que han robado menos. La experiencia delictiva no influye en el sentimiento de culpa en la tarea heteroatribucional, aunque en la autoatribucional aparece un resultado muy significativo teóricamente: puesto que el parámetro beta es negativo, podemos afirmar que el sentimiento de culpa en este contexto varía como función inversa de la experiencia delictiva específica, de modo que los sujetos que más han robado dicen haber sentido menos culpa que los que menos lo han hecho.

Hipótesis 2.4: Las relaciones hipotetizadas más arriba son independientes de la perspectiva de quien juzga.

Los resultados referentes a esta hipótesis se han ido exponiendo conjuntamente con los de las hipótesis anteriores. Claramente, hemos de rechazar la hipótesis de independencia. Excepto en el caso de la relación entre estabilidad causal y predicción, todas las ecuaciones de regresión han variado en función de la perspectiva. 


\section{DISCUSION}

Los resultados perfilan una taxonomía de las causas claramente diferenciada en función de las dimensiones y de la perspectiva de los sujetos.

Las causas del robo, cuando se juzga en general, se consideran internas y controlables, a excepción de la necesidad familiar, la única causa que se considera externa y, por tanto, se conceptualiza como controlable por otros, y no por el agente del robo. En cuanto a la estabilidad, las causas se reparten entre el vicio, las drogas y el tipo de persona - causas estables- y el deseo, la necesidad y la necesidad familiar - causas inestables-. Hasta aquí, nada se aparta del sentido común ni de las respuestas obtenidas en investigaciones anteriores (p.e. Carroll, 1978, 1979).

Cuando, por el contrario, se juzgan las propias acciones, la taxonomía resulta considerablemente distinta: desaparecen las causas estables y la única diferencia significativa que podemos concretar en diferencias de medias es la que se da entre causas internas y causas externas. Aunque aparecen diferencias significativas en el análisis de varianza Kruskal-Wallis con una probabilidad asociada de .03 , no podemos ubicarlas mediante la prueba t de Scheffe al $5 \%$, una prueba conservadora, aunque, de darse, harían referencia a las que menor puntuación en controlabilidad obtienen, es decir, la necesidad y la situación.

Puesto que no se hallaron diferencias significativas en controlabilidad en ninguna de las tareas, podemos considerar que todas las causas del robo ofrecidas por los sujetos en ambas tareas son percibidas o conceptualizadas como controlables, por el sujeto o por otros, dependiendo de si se consideran internas o externas, respectivamente. Quizá una medida más fina de la controlabilidad arrojase alguna diferencia entre las causas autoatribucionales.

Contrastando ambas tareas, el resultado más llamativo es el que se refiere a la necesidad, que se clasifica como más interna en la tarea heteroatribucional que en la autoatribucional. Puesto que necesidad es un término amplio que los sujetos pueden conceptualizar de muy diversa forma en función de los elementos contextuales, podríamos hablar en el futuro de necesidad interna y externa, al igual que en la taxonomía de causas del logro académico se habla de esfuerzo estable y esfuerzo inestable (p.e. Weiner, 1980).

Tampoco es éste un resultado ajeno a la teoría de Weiner y sus colaborado: res, puesto que la clasificación dimensional no es independiente del contexto. Ya Valle y Frieze (1976) habían encontrado que los vendedores a domicilio clasificaban la dificultad de la tarea, típicamente estable en el contexto del logro, como una causa inestable. Respecto de la dicotomía éxito-fracaso, Anderson (1983) encontró que el éxito y el fracaso se percibían más internos y estables en tareas sociales que en las de logro, y Wong y Weiner (1981) encontraron que, a veces, los sujetos mostraban preferencia por ciertas posiciones dimensionales. Betancourt y Weiner (1982) habían investigado sobre este problema con sujetos chilenos y norteamericanos y encontraron una estructura dimensional similar, aunque los chilenos percibieron las causas como más externas, menos estables, y menos controlables que los norteamericanos. No es de extrañar, entonces, que aparezcan estas diferencias en función del escenario de los hechos.

La hipótesis más fuerte del modelo de Weiner en todas sus versiones es la relacionada con la influencia de la estabilidad de las causas sobre las expectativas. En este caso pudimos aceptarla para ambas tareas, aunque explicó una parte considerablemente mayor de la varianza en la tarea heteroatribucional. 
Por otro lado, la hipótesis más novedosa del modelo de Weiner, la que tiene que ver con las emociones, resultó ser la que más débilmente mostró sus efectos. En el caso de los otros, se infirió la existecia de menos sentimiento de culpa cuando se les consideró culpables - ¿verdaderamente culpables?-, en el sentido que uno le da al concepto al juzgarse a sí mismo, cuando la causa es interna y controlable. Con respecto a la tarea autoatribucional, bastó con que la causa fuera controlable, por uno mismo o por otros, para que se sintiera culpa. Parece ser que es algo similar lo que ocurre en el caso de los accidentes: McGraw (1987) interpretó un resultado análogo a éste como indicador de que los sujetos normalmente socializados sienten culpa por las transgresiones accidentales porque, dado que es posible anticipar el sentimiento de culpa, si un sujeto transgrede la norma intencionalmente habrá buscado antes una justificación o, de no tenerla, no cometerá ese acto. No creemos descabellado pensar, por analogía, que el adolescente medio, conociendo como cualquier sujeto normalmente socializado, lo aversivo de tal experiencia, crea que quienes causan intencional o controladamente hechos social y legalmente castigados sientan menos esa culpabilidad. De cualquier forma, a la vista de los resultados, sólo podemos constatar tal relación y justificar su razonabilidad. Sería necesario realizar investigaciones experimentales para operacionalizar el conjunto de variables implicadas.

La perspectiva hizo variar todas las relaciones, excepto la existente entre estabilidad y predicción de reincidencia, lo que indica que el efecto actorobservador está interfiriendo en la operacionalización de las variables, por lo que no se deberían utilizar indistintamente las tareas hetero- y autoatribucional, ni considerarse comparables sus resultados, al menos en las investigaciones relacionadas con la emoción.

De interés criminológico puede ser el resultado que indica que el sentimiento de culpa varía como función inversa de la experiencia delictiva específica, de modo que los sujetos que más han delinquido dicen haber sentido menos culpa, cuando la causa era controlable, que los que no han robado. Además, los sujetos con mayor experiencia delictiva predicen mayor reincidencia en el caso de los otros y en el suyo propio. $\mathrm{Si}$, como a menudo se afirma, la intención puede condiderarse una autopredicción, parece que los delincuentes autodeclarados tienen intenciones de proseguir su carrera delictiva. Investigar con técnicas más sofisticadas el alcance de esta relación y de la que se da entre la experiencia delictiva específica y el sentimento de culpa parece ser una línea de trabajo prometedora, puesto que la investigación de campo, con instrumentos de muy corto alcance, ha podido captar relaciones pequeñas pero significativas.

\section{Referencias}

ANDERSON, C. A. (1983). The causal structure of situations: The generation of plausible causal attributions as a function of the type of event situation. Joumal of Experimental Social Psychology, 19, 185-203.

Ardilla, R. (1986). Etica, psicología y tecnología. Revista de Psicología General y Aplicada, $41,215-239$.

Armon.Jones, C. (1986). The social functions of emotion. En R. Harré (ed.) The social construction of emotions. Oxford, Basil Blackwell.

BANDURA, A. y McDONALD, F. J. (1963). Influence of social reinforcement and the behavior of models in shaping children's moral judgments. Joumal of Abnormal Social Psychology, $67,274-281$. 
Betancourt, H. y Weiner, B. (1982). Attributions for achievement-related events, expectancy and sentiments: a study of success and failure in Chile and the Unites States. Joumal of Cross-cultural Psychology, 13, 362-374.

Carroll, J. S. (1978). Causal attributions in expert parole decisions. Joumal of Personality and Social Psychology, 36, 1.501-1.511.

Carroll, J. S. (1979). Judgments made by parole boards. En I. H. Frieze, D. Bar-Taly J. S. Carroll (eds.) New approaches to social problems: applications of attribution theory. San Francisco, Jossey-Bass.

Carroll, J. S. y PaYNe, J. W. (1976). The psychology of the parole decision process: a joint application of attribution theory and information-processing psychology. En J. S. Carroll y J. W. Payne (eds.) Cognition and social bebavior. Hillsdale, Erlbaum.

Carroll, J. S. Payne, J. W. (1977a). Judgments about crime and the criminal.: a model and a method for investigating parole decisions. En B. D. Sales (ed.) Perspectives in law and psychology, vol. 1: Criminal justice system. Nueva York, Plenum.

Carroll, J. S. y Payne, J. W. (1977b). Crime seriousness, recidivism risk, and causal attribution in judgments of prison term by students and experts. Joumal of Applied Psychology, 62, 595-602.

CRESPO, E. (1986). A regional variation: emotions in Spain. En R. Harré (ed.) The social construction of emotions. Oxford, Basil Blackwell.

DeRivera, J. (1984). The structure of emotional relationships. Review of Personality and Social Psychology, 5, 116-145.

FriJdA, N. H. (1988). The Laws of Emotion. American Psychologist, 43, 349.358.

IZARD, C. E. (1977). Human emotion. New York, Plenum Press.

Izard, C. E. y Saxton, P. M. (1988). Emotions. En R. C. Atkinson, R. J. Herrnstein, G. Lindzey y R. D. Luce (eds.). Steven's Handbook of Experimental Psychology, vol. 1, Nueva York, Wiley.

JONES, E. E. y NisBETT, R. E. (1972). The actor and the observer: divergent perceptions of the causes of behavior. En E. E. Jones, D. E. Kanouse, H. H. Kelley, R. E. Nisbett, S. Valins y B. Weiner (eds.). Attribution: Perceiving the causes of bebavior. Morristown, General Learning Press.

Kelley, H. H. (1972). Causal schemata and the attribution process. En E. E. Jones, D. E. Kanouse, H. H. Kelley, R. E. Nisbett, S. Valins, y B. Weiner (eds.). Attribution: Perceiving the causes of behavior (151-174). Morristown, General Learning Press, 1972.

KuRTINES, W. M. (1986). Moral behavior as rule governed behavior: person and situation effects on moral decision making. Joumal of Personality and Social Psychology, 50, 784-791.

McGraw, K. M. (1987). Guilt following transgression: an attribution of responsibility approach. Joumal of Personality and Social Psychology, 53, 247-256.

Mirón, L.; Otero, J. M. y Luengo, A. (1989). Empatía y conducta antisocial. Comunicación presentada en el Congreso Nacional de Psicología Social. En prensa.

Moreno.Jiménez, B. (1986). Emotion end attribution. Revista de Psicología Social, 1, 71-78.

KuSSELL, D. (1982). The causal dimension scaie: a measure of how individuals perceive causes. Joumal of Personality and Social Psychology, 42, 1.137-1.145.

Russell, D. W.; MCAuley, E. y TARICO, V. (1987). Measuring causal attributions for success and failure: a comparison of methodologies for assessing causal dimensions. Joumal of Personality and Social Psychology, 52, 1248-1257.

SABIN1, J. y SILver, M. (1982). Moralities of Everiday Life. Londres, Oxford University Press.

SALGADO, J. (1987). Investigaciones sobre atribución: las dimensiones atribucionales. Revista de Psicología Social, 2, 43-49.

Scherer, K. R. y Tannenbaum, P. H. (1986). Emotional experiences in everyday life: a survey approach. Motivation and Emotion, 10, 295-314.

Shaver, K. G. (1985). The attribution of blame. Nueva York, Springer-Verlag.

Shaver, K. G.; Null, C. H., y HufF, C. W. (1982). Multidimensional scaling of responsibility related words. Ponencia presentada en la reunión de la Psychonomic Society, Minneapolis.

VALLE, V. A. y FrIEZE, I. H. (1976). Stability of causal attributions as a mediator in the changing of expectations for success. Journal of Personality and Social Psychology, 33, 579.587.

WeInER, B. (1980). Human Motivation. Nueva York, Springer-Verlag.

WEINER, B. (1986). An attributional theory of motivation and emotion. Nueva York: SpringerVerlag.

WEINER, B. (1987). The role of emotions in a theory of motivation. En F. Halisch y J. Kuhl. Motivation, Intention and Volition. Berlín, Springer-Verlag.

Weiner, B.; Graham, S. y Chandler, C. C. (1982). Pity, anger and guilt: An attributional analysis. Personality and Social Psychology Bulletin, 8, 226-232.

Weiner, B.; Russell, D. y Lerman, D. (1978). Affective consequences of causal ascriptions. En J. H. Harvey, W. Ickes y R. F. Kidd (eds.) New directions in attribution research, vol. 2, Hillsdale, Lawrence Erlbaum.

Weiner, B.; RusSeLl, D. y LeRMAN, D. (1979). The cognition-emotion process in achievementrelated contexts. Journal of Personality and Social Psycbology, 37, 1.121-1.220.

WONG, P. T. P. y WEINER, B. (1981). When people ask «why" questions and the heuristics of attributional search. Joumal of Personality and Social Psychology, 40, 650-663. 